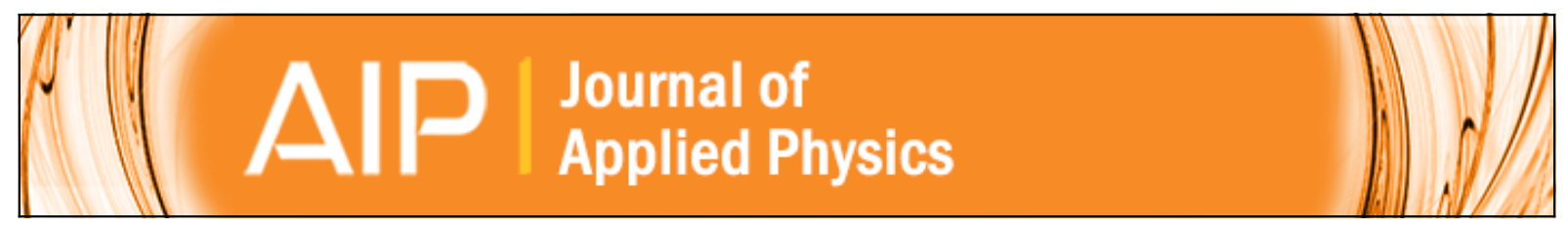

\title{
Photonic crystals with defect structures fabricated through a combination of holographic lithography and two-photon lithography
}

Kris Ohlinger, Faraon Torres, Yuankun Lin, Karen Lozano, Di Xu, and Kevin P. Chen

Citation: Journal of Applied Physics 108, 073113 (2010); doi: 10.1063/1.3493119

View online: http://dx.doi.org/10.1063/1.3493119

View Table of Contents: http://scitation.aip.org/content/aip/journal/jap/108/7?ver=pdfcov

Published by the AIP Publishing

\section{Articles you may be interested in}

Two-photon fabrication of photonic crystals by single-beam laser holographic lithography

J. Appl. Phys. 107, 074311 (2010); 10.1063/1.3374476

Fabrication of two-dimensional coupled photonic crystal resonator arrays by holographic lithography

Appl. Phys. Lett. 89, 041902 (2006); 10.1063/1.2234743

Fabrication of hetero-binary and honeycomb photonic crystals by one-step holographic lithography

Appl. Phys. Lett. 88, 091115 (2006); 10.1063/1.2181270

Fabrication of large area two- and three-dimensional polymer photonic crystals using single refracting prism holographic lithography

Appl. Phys. Lett. 86, 241102 (2005); 10.1063/1.1947369

Three-dimensional photonic crystals fabricated by visible light holographic lithography

Appl. Phys. Lett. 82, 2212 (2003); 10.1063/1.1565682

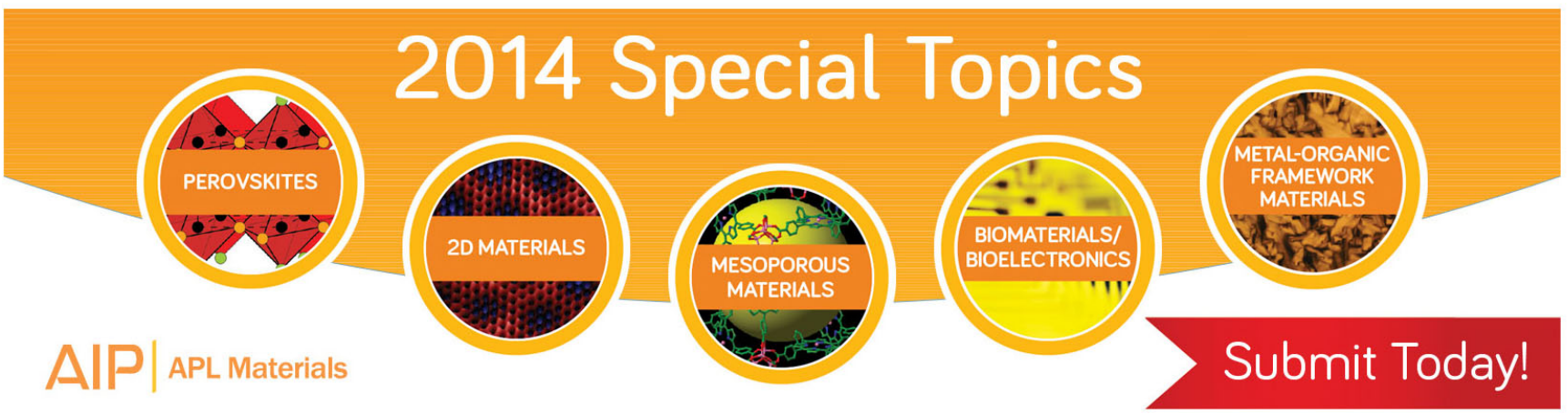




\title{
Photonic crystals with defect structures fabricated through a combination of holographic lithography and two-photon lithography
}

\author{
Kris Ohlinger, ${ }_{3}^{1}$ Faraon Torres, ${ }^{1}$ Yuankun Lin, ${ }^{1, a)}$ Karen Lozano, ${ }^{2} \mathrm{Di} \mathrm{Xu},{ }^{3}$ and \\ Kevin P. Chen ${ }^{3}$ \\ ${ }^{1}$ Department of Physics and Geology, University of Texas-Pan American, Edinburg, Texas 78539, USA \\ ${ }^{2}$ Department of Mechanical Engineering, University of Texas-Pan American, Edinburg, Texas 78539, USA \\ ${ }^{3}$ Department of Electrical and Computer Engineering, University of Pittsburgh, Pittsburgh, \\ Pennsylvania 15261, USA
}

(Received 21 July 2010; accepted 23 August 2010; published online 8 October 2010)

\begin{abstract}
This paper presents the capability of direct laser writing of complex defect structures in holographically formed three-dimensional photonic crystals in dipentaerythritol penta/hexaacrylate (DPHPA) monomers mixed with photoinitiators. The three-dimensional photonic crystal template was fabricated through prism-based holographic lithography. Defect structures are fabricated through the two-photon polymerization excited by a femtosecond laser. The strengths of two optical lithographic techniques are combined with holographic lithography providing a rapid and large area microfabrication and two-photon lithography providing flexibility in fabrication of defect structures. The optical fabrication process is simplified in the negative tone DPHPA without prebake and postexposure bake as is required of SU-8 while maintaining a capability for constructing photonic structures with small features. (0) 2010 American Institute of Physics. [doi:10.1063/1.3493119]
\end{abstract}

\section{INTRODUCTION}

Photonic crystals $(\mathrm{PhCs})$ are micro/nanostructured materials in which the dielectric constant is periodically modulated on a length scale comparable to the desired wavelength of operation. ${ }^{1}$ In PhCs, a set of wavelengths are forbidden to propagate in the crystal, i.e., a photonic bandgap exists, similar to the electronic bandgap in semiconductors. In the last decade, several elegant techniques have succeeded in fabricating three-dimensional (3D) $\mathrm{PhCs}$, such as conventional multilayer stacking of woodpile structures using semiconductor fabrication processes,${ }^{2}$ colloidal self-assembly, ${ }^{3}$ multiphoton direct laser writing, ${ }^{4}$ and holographic lithography. ${ }^{5}$ The semiconductor processing techniques provide advanced tools for building structure layer-by-layer and incorporating defects at any depth. ${ }^{2}$ Most of the direct laser writing was based on two-photon polymerization of photosensitive resins. When a high-intensity light shines on a material, the probability for two-photon absorption is proportional to the square of the optical field intensity, and thus is greatest at the center of a Gaussian laser beam. When tightly focused into the volume of a photosensitive resin, the polymerization process can be initiated by nonlinear absorption of near infrared femtosecond laser pulses within the focal volume. By moving the laser focus three-dimensionally through the resin, the two-photon polymerization happens at the focus points. ${ }^{4} 3 \mathrm{D}$ structures and defects such as missing rod in woodpile structure can be fabricated by a computer-aided sample stage control. $^{4,6}$ The PhC structures fabricated by above two approaches are dedicated but the process are time-consuming. Fabrication of defects in self-assembled opal $\mathrm{PhC}$ has made headway by surface modification followed by overgrowth of

\footnotetext{
${ }^{a)}$ Electronic mail: yuankun.lin@unt.edu. Current address: Department of Physics, University of North Texas, Denton, Texas 76203, USA.
}

$\mathrm{opal}^{7}$ or laser direct writing of defect structures within the opal. ${ }^{8,9}$ However, opal PhCs were limited to face-centeredcubic structures with a relative small photonic bandgap.

Multibeam interference-based holographic lithography has been successful for fabricating 3D PhCs by exposing a photoresist or polymerizable resin to interference patterns of laser beams. ${ }^{5,10-17}$ In the case of positive resist, the overexposed material is then dissolved away in the post-exposure processing. The underexposed region forms a periodic network and acts as a 3D PhC template. For negative photoresists, the underexposed regions can then be selectively removed using a developer while overexposed region becomes polymerized and forms a periodic network for PhC template. Multiple-beam holographic lithography was complicated when it was based on multiple bulk optical elements such as mirrors and beam splitters. ${ }^{5}$ The optical setup for holographic lithography has been simplified by using a single optical element such as flat-top prism ${ }^{17}$ or a phase mask..$^{12,13}$ Multilayer phase masks ${ }^{14}$ have been demonstrated for the fabrication of $\mathrm{PhC}$ templates by introducing a phase difference among the diffracted beams through changing the distance between two orthogonally oriented gratings. These single-beam and single-exposure processes using the multilayer mask in holographic lithography have drastically reduced the fabrication complexity. To be useful for optoelectronics applications, the incorporation of engineered defects must be realized in PhCs. A significant advance has been achieved on the simultaneous fabrication of functional defects in PhC templates by using a multibeam phasecontrolled one-step holographic lithography ${ }^{15}$ and by combining the amplitude mask with the phase mask. ${ }^{16}$ However, the process still needs to be improved to increase the uniformity of the samples.

The strength of holographic lithography and two-photon lithography can be combined by using the holographic li- 


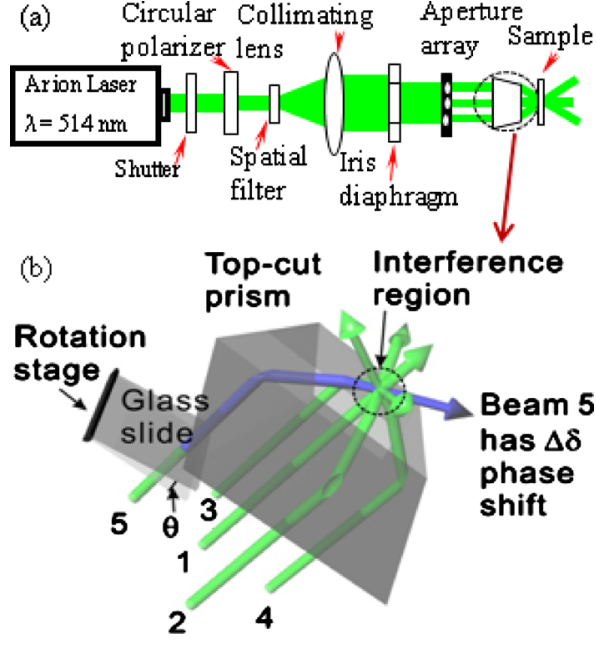

FIG. 1. (Color online) (a) Experimental setup for laser holographic lithography; (b) scheme of five-beam interference produced through a flat-top four-side prism. The phase delay is introduced via the inserted glass slide.

thography for a rapid and flexible fabrication of 3D host PhCs and using the two-photon lithography for creating desired defects in $\mathrm{PhCs}$, that in principle, can be placed in any depth with any shape. Two-photon ablation or polymerization was used to create defects in PhC template in SU-8, which were created through holographic lithography, by three research groups in $2005,{ }^{18} 2006,{ }^{19}$ and $2008 .^{20}$ Sun et $a l .{ }^{18}$ demonstrated the hybrid holographic and direct writing method in a two-dimensionally periodic structure while Scrimgeour et al. ${ }^{19}$ described the creation of defect structures inside 3D PhCs. The other group ${ }^{20}$ fabricated and developed polymer-air PhCs in SU-8 via holographic lithography and the $\mathrm{PhCs}$ were infiltrated with trimethylol propane triacrylate and a two-photon sensitive photoinitiator for the direct writing of defects via an ultrafast laser. Nice defects with a size of several lattice constants were demonstrated. These three groups used SU-8 photoresist for the holographic fabrication. The SU-8 usually requires prebake and postexposure bake although it is an excellent resist for fabricating nano/ mcrostructures.

In this paper, we use a different photosensitive mixture of dipentaerythritol penta/hexaacrylate (DPHPA) and photoinitiator for the hybrid holographic and two-photon lithography without prebake and postexposure bake. A phase-tunable single optical element is used for the holographic fabrication of large area woodpile-type $\mathrm{PhC}$ template in order to reduce the optics setup complexity. The two-photon lithography is demonstrated in the same photosensitive mixture without an infiltration of other photosensitive mixture. We create complex defect structures including letters through direct writing in the $3 \mathrm{D}$ holographically formed $\mathrm{PhC}$ template.

\section{FABRICATION OF 3D PHOTONIC STRUCTURES THROUGH HOLOGRAPHIC LITHOGRAPHY}

A $514.5 \mathrm{~nm}$ laser beam from a Sabre Ar ion laser (Coherent Inc.) was used for the five-beam interference based holography lithography. The laser beam was circularly polarized, cleaned, expanded, and collimated by spatial filter and collimating lens set as shown in Fig. 1(a). As shown in detail in Fig. 1(b), the single optical element used to construct fivebeam interference pattern is a top-cut four-side-prism. ${ }^{17}$ After total-internally reflected at the four lateral surfaces, the beam is separated into five beams with centrosymmetric propagation directions. After they refracted through the prism surface, all five beams were recombined to create a $3 \mathrm{D}$ interference region behind the prism surface. A thin microscope glass cover slide mounted on a rotatable stage is inserted into one of the five beams to control the phase of the beam for the generation of 3D interconnected PhCs. ${ }^{17}$ When the relative phase shift in one beam is $\pi$, the 3D interference pattern has a diamondlike structure (or woodpile). ${ }^{11} 3 \mathrm{D}$ photonic structures were fabricated holographically in a negative tone photoresist. The visible light sensitive photoresist contains 88.80 wt \% DPHPA monomer (from Aldrich), 0.11 wt $\%$ photoinitiator rose Bengal, 0.79 wt $\%$ coinitiator $\mathrm{N}$-phenyl glycine, and 10.30 wt \% chain extender $\mathrm{N}$-vinyl pyrrolidinone. The photosensitive mixture was spin-coated as a thin film on a cut glass slide. The spin-coating speed was between 800 and $2000 \mathrm{rpm}$ and the sample thickness was between 6 and $18 \mu \mathrm{m}$. A laser power between 150 and 300 $\mathrm{mW}$ was used. The exposure time was several seconds. After the exposure, the sample was developed in propylene glycol methyl ether acetate for 6 min typically. More development times than 6 min were applied for thick samples and a magnetic bar was used to stir the propylene glycol methyl ether acetate developer with a speed of $60 \mathrm{rpm}$. Finally the sample was washed by isopropanol for $20 \mathrm{~s}$ and left to dry in ambient air. The developed holographic sample has a surface area of $3.5 \times 3.5 \mathrm{~mm}^{2}$.

Figure 2(a) shows a scanning electron microscope (SEM) image of photonic structures developed in the photoresist, showing the quality of woodpile structure fabricated through holographic lithography. The cross-section of the fabricated sample is shown in Fig. 2(b) and an enlarged view is shown in Fig. 2(c). The photonic structure is determined by the wave vector $k$ of the five beams as described by Eqs. (1) and (2),

$$
\begin{aligned}
& k_{0}=K(0,0,1), \\
& k_{n}=K\left(\sin \alpha \cos \frac{4 n \pi}{8}, \sin \alpha \sin \frac{4 n \pi}{8}, \cos \alpha\right),
\end{aligned}
$$$$
\text { for } n=1-4 \text {, }
$$

where $\alpha$ is the interference angle between the side-beam $k_{n}$ and the central-beam $k_{0}$. The wave vector difference $\Delta k$ can be considered as reciprocal vectors of holographically formed structures. The periodicity in $x$ or $y$ direction is $\lambda / \sin \alpha$ which is calculated to $0.84 \mu \mathrm{m}$ for $\alpha=37.5^{\circ}$. The periodicity is measured to be $0.83 \mu \mathrm{m}$ averaged over several SEMs. The periodicity in $z$-direction is $\lambda_{m} /\left(1-\cos \alpha_{m}\right)$ $=4.21 \mu \mathrm{m}$ assuming the refractive index is 1.58 for the photoresist (subscript $\mathrm{m}$ for value inside the mixture). The measured value is $3.96 \mu \mathrm{m}$ which is smaller than theory. The $6 \%$ difference is due to the sample shrinkage in DPHPA, while a shrinkage of $16 \%$ has been reported in SU-8 PhC template. ${ }^{21}$ The wavelike long period banding in the surface of the structure in Figs. 2(a) and 2(b) is caused by a small 


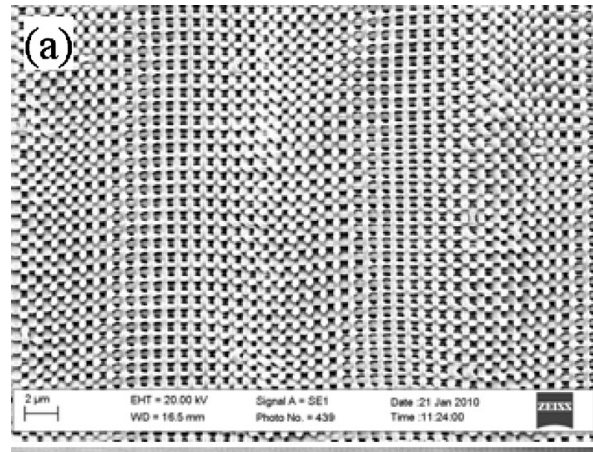

(b)
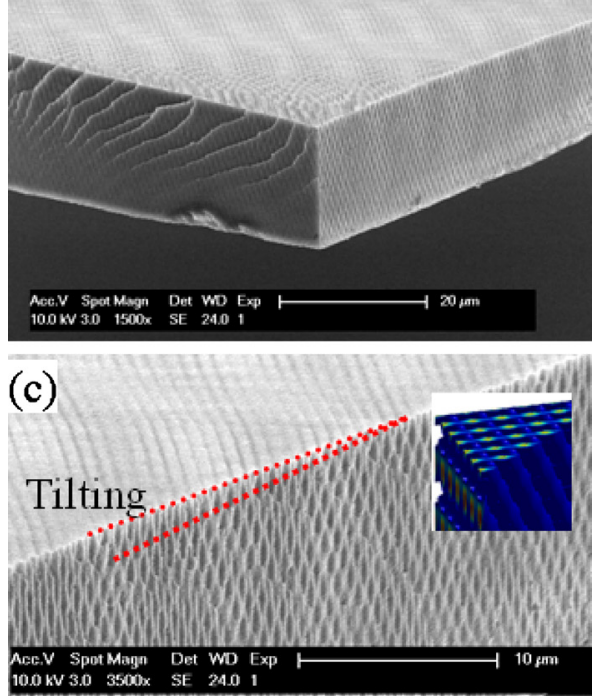

FIG. 2. (Color online) (a) SEM of fabricated 3D PhC template through holographic lithography; (b) cross-section SEM image of the fabricated $\mathrm{PhC}$; (c) enlarged view of the cross-section. The insert shows a simulated five-beam interference pattern.

sample tilt as illustrated in Fig. 2(c). The cross-section of the sample also shows a nonuniformity. It happens when the sample is not cut along the $x$ or $y$-axis, or along the diagonal direction as shown in the simulated figure inserted in Fig. 2(c). When the elliptical rods are close to the joint section of rods (polymerized under high laser intensity), their crosssection is bigger than those rods polymerized under low laser intensity.

\section{TWO-PHOTON POLYMERIZATION THROUGH LASER DIRECT WRITING}

Figure 3(a) shows the laser direct writing setup. A tunable Ti:sapphire femtosecond laser (Chameleon, Coherent Inc.) was used with a repetition rate of $80 \mathrm{MHz}$ and pulse width of $140 \mathrm{fs}$. The laser operation wavelength is $720 \mathrm{~nm}$. The laser beam was controlled through an external shutter and the laser power was attenuated through neutral density filters. The laser beam was expanded and then focused inside the photoresist using a $0.55 \mathrm{NA}$ aspheric lens (Thorlabs). The laser power was $150 \mathrm{~mW}$ as measured before the aspheric lens. The photoresist was spin-coated on a glass slide and the slide was mounted on a three axial motion stage (Newport) which was controlled with a computer and translated with a constant speed of $2.4 \mathrm{~mm} / \mathrm{s}$. The same photoresist mixture

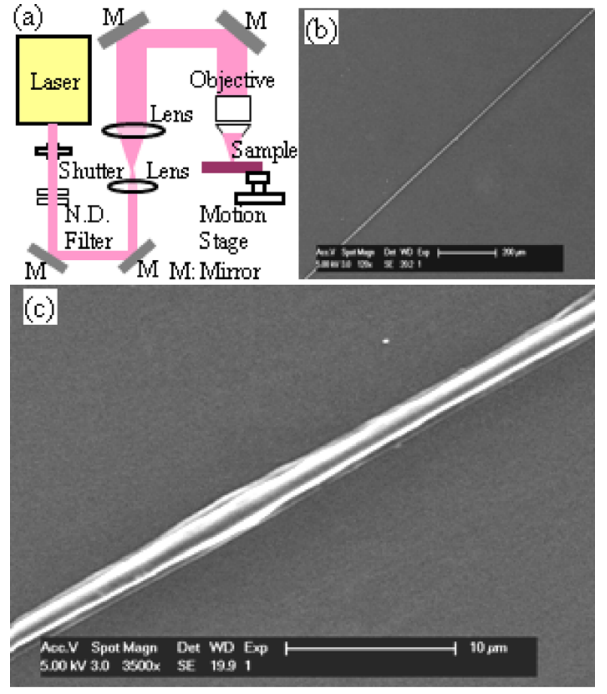

(d)

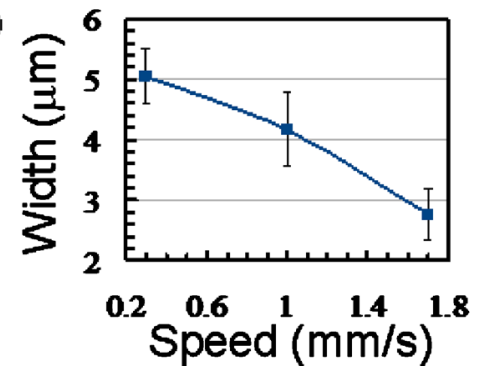

FIG. 3. (Color online) (a) Experimental setup for two-photon lithography; (b) large area SEM image of the polymer line on the glass substrate; (c) enlarged SEM view of the polymer line on glass substrate; (d) line width of polymer structures fabricated at three scanning speeds.

and spin-coating on the glass slide were used as described in Sec. II. After the laser direct writing, the photoresist was developed in propylene glycol methyl ether acetate and then washed by isopropanol. Figure 3(b) shows a large area SEM image of the direct laser written polymer line structure standing on the glass slide substrate using the two-photon lithography method, showing that the two-photon polymerization can be realized in the DPHPA mixture. Figure 3(c) shows an enlarged SEM image of the fabricated line structure on the glass substrate. From the figure, we can see that the polymer line has clean surface and a width of $2.3 \mu \mathrm{m}$. The profile of the polymerized line can be modified by using an aspheric lens with big numeric aperture. The variation in the line width can be reduced by improving the mechanical stability of the motion stages and the steadiness of the laser output power. Figure 3(d) shows the change in polymer line width for three scanning speeds. The data were collected from polymer lines on the same glass slide substrate under the same laser power of $100 \mathrm{~mW}$ so that experimental conditions were same except the laser scanning speed. As seen from Fig. 3(d), when the scanning speed increases, the line width decreases.

\section{FABRICATION DEFECT STRUCTURES IN HOLOGRAPHIC PHCS}

The strength of above optical lithography techniques can be utilized for the fabrication of 3D PhCs with defects. As a demonstration, Fig. 4 shows defect structures fabricated in 

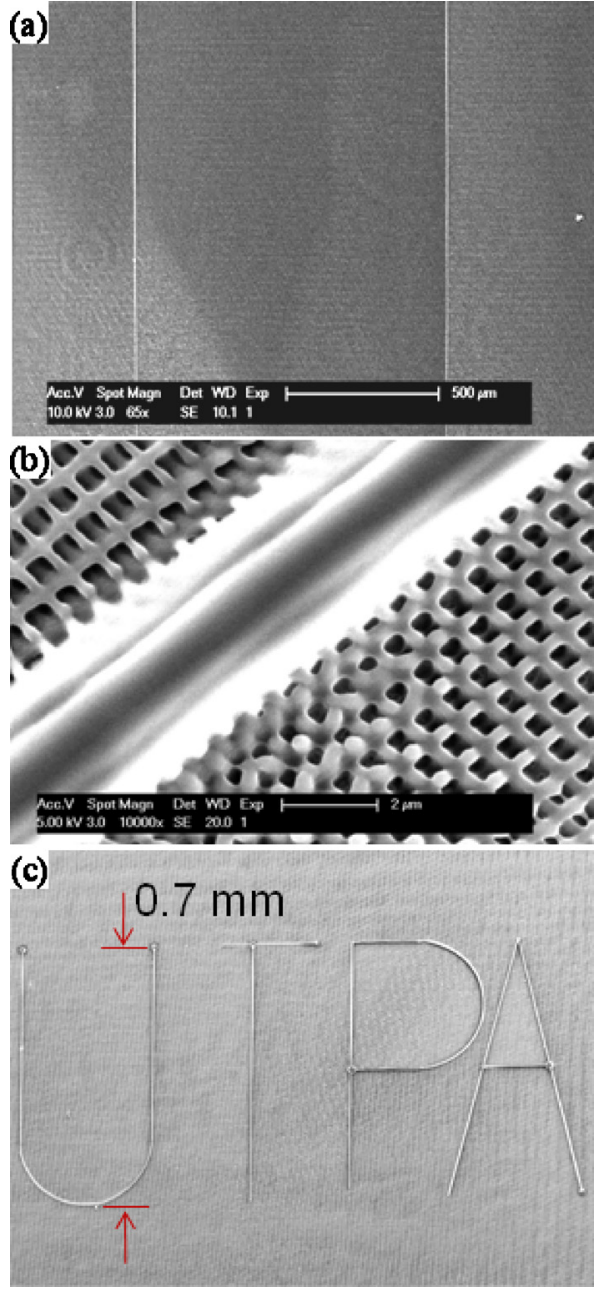

FIG. 4. (Color online) (a) Large area SEM image of defect lines fabricated in holographic $\mathrm{PhC}$ template; (b) an enlarged view of SEM image of defect line in 3D PhC template; (c) defect structures in UTPA letters are fabricated in $3 \mathrm{D} \mathrm{PhC}$ template. The height of letters is $0.7 \mathrm{~mm}$ as designed in the motion stage control program.

the 3D PhC template by combining the above two opticallithography methods. The spin-coated photoresist film was first exposed to the Ar ion laser for the holographic lithography. After initial exposure, the glass slide is then mounted on the computer controlled motion stage. Then the femtosecond laser pulses were focused inside the previously holographically exposed resist. The motion stage was controlled such that the focal point moves across the undeveloped $\mathrm{PhC}$ region. The same motion speed of $2.4 \mathrm{~mm} / \mathrm{s}$ was used. After exposure, the photoresist was developed in propylene glycol methyl ether acetate for $6 \mathrm{~min}$ and then washed with isopropanol for $20 \mathrm{~s}$ and left to dry in ambient air. Figure 4(a) shows the large scale uniformity while Fig. 4(b) shows the interfacial quality of the two lithography processes. The width of the defect line is approximately $3 \mu \mathrm{m}$. A complicated structure can also be fabricated with the two-photon lithography. Figure 4(c) shows defect structures of UTPA (University of Texas-Pan American) letters fabricated in the 3D PhC template, which clearly demonstrates the capability of direct laser writing of complex structure through the twophoton lithography in holographic structures. The scale bar was missing when taking SEM. The height of letters was 0.7 $\mathrm{mm}$ and the distance between letter $\mathrm{U}$ and letter $\mathrm{A}$ was 1.478 $\mathrm{mm}$ as designed by the motion stage control program.

\section{CONCLUSIONS}

In summary, this paper demonstrated the capability of optical fabrications of PhCs with defect structures in DPHPA instead of SU-8 through a simplified lithographic process without prebake and postexposure bake. The flat-top fourside prism-based holographic lithography was used for the rapid fabrication of large area $3 \mathrm{D} \mathrm{PhC}$ templates with woodpile-type structure. Two-photon lithography was capable to write complex defect structures in the holographically formed 3D PhCs in DPHPA with a clear interfacial connection. Thus DPHPA mixture can be used as photoresist for holographic lithography, laser direct-writing, and hybrid holographic and two-photon lithography for constructing photonic nano/microstructures.

\section{ACKNOWLEDGMENTS}

This work is supported by research grants from the U.S. National Science Foundation under Grant Nos. DMI0609345, CMMI-0900458, CMMI-0900564 and the U.S. Air Force Grant No. FA9550-06-1-0518. We thank Dr. Anxiu Kuang for her SEM support.

${ }^{1}$ J. D. Joannopoulos, P. R. Villeneuve, and S. H. Fan, Nature (London) 386, 143 (1997).

${ }^{2}$ M. Ho, C. T. Chan, C. M. Soukoulis, R. Biswas, and M. Sigalas, Solid State Commun. 89, 413 (1994).

${ }^{3}$ A. Blanco, E. Chomski, S. Grabtchak, M. Ibisate, S. John, S. W. Leonard, C. Lopez, F. Meseguer, H. Miguez, J. P. Mondia, P. Jessica, G. A. Ozin, A. Geoffrey, O. Toader, and H. M. van Driel, Nature (London) 405, 437 (2000).

${ }^{4}$ M. Deubel, G. V. Freymann, M. Wegener, S. Pereira, K. Busch, and C. M. Soukoulis, Nature Mater. 3, 444 (2004).

${ }^{5}$ M. Campbell, D. N. Sharp, M. T. Harrison, R. G. Denning, and A. J. Turberfield, Nature (London) 404, 53 (2000).

${ }^{6}$ H.-B. Sun, V. Mizeikis, Y. Xu, S. Juodkazis, J.-Y. Ye, S. Matsuo, and H. Misawa, Appl. Phys. Lett. 79, 1 (2001).

${ }^{7}$ E. Vekris, V. Kitaev, G. von Freymann, D. D. Perovic, J. S. Aitchison, and G. A. Ozin, Adv. Mater. 17, 1269 (2005).

${ }^{8}$ W. M. Lee, S. A. Pruzinsky, and P. V. Braun, Adv. Mater. 14, 271 (2002).

${ }^{9}$ Y. Jun, C. A. Leatherdale, and D. J. Norris, Adv. Mater. 17, 1908 (2005).

${ }^{10}$ S. Yang, M. Megens, J. Aizenberg, P. Wiltzius, P. M. Chaikin, and W. B. Russel, Chem. Mater. 14, 2831 (2002).

${ }^{11}$ O. Toader, T. Y. M. Chan, and S. John, Appl. Phys. Lett. 89, 101117 (2006).

${ }^{12}$ I. Divliansky, T. S. Mayer, K. S. Holliday, and V. H. Crespi, Appl. Phys. Lett. 82, 1667 (2003).

${ }^{13}$ Y. Lin, P. R. Herman, and K. Darmawikarta, Appl. Phys. Lett. 86, 071117 (2005).

${ }^{14}$ Y. Lin, A. Harb, D. Rodriguez, K. Lozano, D. Xu, and K. P. Chen, Opt. Express 16, 9165 (2008).

${ }^{15}$ J. Li, Y. Liu, X. Xie, P. Zhang, B. Liang, L. Yan, J. Zhou, G. Kurizki, D. Jacobs, K. S. Wong, and Y. Zhong, Opt. Express 16, 12899 (2008).

${ }^{16}$ Y. Lin, A. Harb, K. Lozano, D. Xu, and K. P. Chen, Opt. Express 17, 16625 (2009).

${ }^{17}$ Di Xu, K. P. Chen, A. Harb, D. Rodriguez, K. Lozano, and Y. Lin, Appl. Phys. Lett. 94, 231116 (2009).

${ }^{18}$ H. Sun, A. Nakamura, K. Kaneko, S. Shoji, and S. Kawata, Opt. Lett. 30, 881 (2005).

${ }^{19}$ J. Scrimgeour, D. N. Sharp, C. F. Blanford, O. M. Roche, R. G. Denning, and A. J. Turberfield, Adv. Mater. 18, 1557 (2006).

${ }^{20}$ V. Ramanan, E. Nelson, A. Brzezinski, P. V. Braun, and P. Wiltzius, Appl. Phys. Lett. 92, 173304 (2008).

${ }^{21}$ D. C. Meisel, M. Diem, M. Deubel, F. Perez-Willard, S. Linden, D. Gerthsen, K. Busch, and M. Wegener, Adv. Mater. 18, 2964 (2006). 